\title{
Representaciones sociales de los métodos anticonceptivos
}

Social Representations of Contraceptive Methods

Representações sociais de métodos contraceptivos

María del Carmen Beltrán Montenegro

Escuela Superior de Enfermería Culiacán, Universidad Autónoma de Sinaloa, México

beltrancarmelita@hotmail.com

Jesús Roberto Garay Núñez

Escuela Superior de Enfermería Culiacán, Universidad Autónoma de Sinaloa, México

jrgarayn@hotmail.com

\section{Resumen}

En México, $64 \%$ de las mujeres en edad reproductiva de 15 a 19 años con vida sexual activa, no usan métodos anticonceptivos. Seis de cada diez adolescentes que regularon su fecundidad recurren a los métodos del ritmo y retiro. Asimismo, $34 \%$ de los adolescentes ha utilizado un método anticonceptivo durante la primera relación sexual. Los índices de mortalidad materna en mujeres de 15 a 19 años de edad son el doble de los correspondientes a las mujeres de 20 a 29 años. Por ello es de suma importancia conocer las representaciones sociales de los métodos anticonceptivos en estudiantes de una universidad pública, de la ciudad de Culiacán, Sinaloa, para determinar el núcleo central de la representación y establecer estrategias innovadoras de educación sexual y reproductiva y así prevenir embarazos no deseados y enfermedades de transmisión sexual. La metodología utilizada fue el paradigma cualitativo interpretativo de orientación procesual.

Palabras clave: representaciones sociales, métodos anticonceptivos. 


\section{Abstract}

In Mexico, 64\% of women in reproductive age of 15 to 19 years with sexually active, do not use contraceptive methods. Six of every ten teenagers which regulated their fertility resort to the Rhythm and Pull Out Method (Withdrawal) methods. In addition, 34\% of teens been used a method of birth control during sexual intercourse. The rates of maternal mortality in women of 15 to 19 years of age are twice those for women 20 to 29 years. Therefore it is important to know social representations of contraceptive methods in students of a public University in the city of Culiacán, Sinaloa, to determine the central core of the representation and establish innovative sexual and reproductive education strategies and thus prevent unwanted pregnancies and sexually transmitted diseases. The methodology used was the qualitative interpretive paradigm of procedural guidance.

Key Words: social representations, contraceptive methods.

\section{Resumo}

No México, 64\% das mulheres em idade reprodutiva, de 15 a 19 anos sexualmente ativos, não utilizar contracepção. Seis em cada dez adolescentes que regulavam sua resort fertilidade aos métodos de ritmo e de retirada. Além disso, 34\% dos adolescentes têm usado um método contraceptivo na primeira relação sexual. taxas de mortalidade materna em mulheres de 15 a 19 anos são duas vezes aquelas para mulheres com idade entre 20 a 29 anos. Portanto, é extremamente importante conhecer as representações sociais dos métodos anticoncepcionais por estudantes de uma universidade pública, a cidade de Culiacan, Sinaloa, para determinar o núcleo de representação e inovadoras estratégias de educação em saúde sexual e reprodutiva e evitar gravidezes doenças indesejadas e doenças sexualmente transmissíveis. A metodologia utilizada foi a orientação processo interpretativo paradigma qualitativo.

Palavras-chave: representações sociais, contracepção.

Fecha recepción: Enero 2016

Fecha aceptación: Julio 2016 


\section{Introducción}

La atención de la salud sexual de los adolescentes ha sido un tema bastante trabajado en diferentes espacios académicos, políticos y sociales. A pesar de ello, las reflexiones no se agotan, por el contrario se plantean más inquietudes y problemas a resolver. Cuando se especifica que la población adolescente es diversa, no sólo por sus características individuales, sino también por los contextos que los determinan, los problemas y las reflexiones se amplían y tocan diferentes campos del saber (Collazos, 2012). Para ello se exploraron la estructura y los contenidos de las representaciones sociales de los métodos anticonceptivos en dicentes de la escuela de enfermería de una universidad pública en la ciudad de Culiacán, Sinaloa.

En América Latina y el Caribe la población entre los 10 y 24 años de edad alcanzó 155 millones en el año 2000, lo cual representa alrededor de un tercio de la población total de la región. En esta región muchos jóvenes están teniendo relaciones sexuales, a menudo sin protección, lo cual los coloca en riesgo de embarazos no deseados, abortos inseguros, e infecciones de transmisión sexual (ITS), incluyendo el VIH. Las investigaciones revelan que el embarazo adolescente sigue siendo frecuente en América Latina, y que en el Caribe la epidemia del VIH/SIDA es alarmante entre los más jóvenes. Sin embargo, algunas iniciativas para atender las necesidades de salud sexual y reproductiva de los jóvenes están dando resultados alentadores (Moya, 2002).

México es un país profundamente desigual; las prácticas sexuales y reproductivas de sus pobladores responden a un amplio y muy diferenciado abanico de condiciones de vida, mientras sus niveles de pobreza, lo mismo que el rezago en materia de alimentación, educación, derechos sociales, prevención y atención a la salud, inciden de manera directa sobre el estado que guarda su salud sexual y reproductiva (Lerner, 2009).

Las múltiples desigualdades saltan a la vista. Al calificar como un éxito que las mujeres mexicanas estén posponiendo la edad para tener el primer hijo, no suele indicarse que son mayoritariamente las de clase alta las que se convierten en madres a los 24 años, mientras que aquellas que viven en condiciones más precarias lo hacen antes de llegar a los 19 años, 
teniendo un mayor número de hijos en comparación con las primeras. La situación se agudiza entre las mujeres campesinas e indígenas, pues sólo la mitad de ellas recurre a la anticoncepción (Lerner, 2009).

Comprender los desafíos de la SSR desde la perspectiva de los derechos humanos, de la justicia social y la ciudadanía implica reconocer que el Estado mexicano está obligado a incorporar las dimensiones de la desigualdad, fundamentalmente las de género, las económicas, étnicas y generacionales en sus planes y políticas de población, del modo como se comprometió en el Programa de Acción de la IV Conferencia Internacional sobre la Población y el Desarrollo (El Cairo, 1994).

Los comportamientos en el cuidado de la salud están enraizados por los conocimientos acumulados y por las representaciones sociales, que tienen un carácter dinámico y se van construyendo en las diferentes etapas de la vida. A su vez, el mundo simbólico comprende estructuras de pensamiento y marcos de referencia desde los cuales se interpretan los fenómenos que afectan a los sujetos configurando un sistema de pensamiento lógico que es aplicado en las prácticas cotidianas. Cuando el pensamiento lógico está guiado por el mundo simbólico, los fenómenos de la salud y la enfermedad se codifican desde ese marco de referencia, dando lugar a comportamientos específicos que están de acuerdo con su trasfondo explicativo y que resultan muy resistentes al cambio, debido al proceso de objetivación, naturalización y anclaje (Candreva, 2004).

\section{Desarrollo de contenidos sobre la temática seleccionada}

La población de Sinaloa muestra hoy profundas transformaciones que afectan su crecimiento y su estructura por edad. La disminución en la mortalidad infantil, los nuevos patrones de causa de muerte, la mayor esperanza de vida al nacer, el aumento del uso de métodos anticonceptivos modernos y la intensificación de las migraciones son responsables directos de estos cambios, entre otros factores. La fecundidad es uno de los principales componentes del crecimiento de población y del cambio en la estructura por edad. El descenso de la fecundidad en Sinaloa se ha debido principalmente al creciente acceso a los servicios de salud reproductiva, incluyendo la información y disponibilidad de métodos 
anticonceptivos en los servicios de salud. Esto ha permitido a mujeres y hombres planificar mejor sus familias, específicamente sobre el número de hijos que desean tener y el momento en el que los desean, a la vez la disminución de la fecundidad ha contribuido a minimizar los riegos de salud de las mujeres y de los niños (Fernández, P., et al., 2014).

Dentro del grupo de MEF destaca la presencia de adolescentes (15 a 19 años) que requieren atención especial por los riesgos inherentes a estas edades. Los requerimientos se centrarían en generar programas de educación sexual para prevenir enfermedades de transmisión sexual y el síndrome de inmunodeficiencia adquirida (VIH/SIDA), evitar embarazos no planificados y hacer uso adecuado de métodos anticonceptivos, así como el riesgo de consumir drogas. En 2010, las adolescentes representaron un porcentaje importante dentro del grupo de MEF, debido a que alrededor de una de cada seis mujeres (17.5\%) era adolescente. Durante el periodo de proyección se espera pase de $17.2 \%$ en 2013 a $15.9 \%$ en 2020 y a $14.8 \%$ en 2030 (Fernández, P., et al., 2014).

En relación con el conocimiento que los adolescentes sinaloenses tienen de los métodos anticonceptivos, $90.5 \%$ de la población de 12 a 19 años de edad informó haber escuchado hablar de algún método anticonceptivo. Asimismo, la respuesta a esta pregunta por grupos de edad muestra que $85.5 \%$ de los adolescentes de menor edad (12 a 15 años) dijo haber escuchado de algún método, al igual que $94.7 \%$ de los de 16 a 19 años. En contraste, entre los adolescentes de 12 a 19 años de edad, $73.9 \%$ en 2000 y $79.4 \%$ en 2006 declararon conocer o haber escuchado hablar de algún método anticonceptivo (ENSANUT, 2012).

Por otra parte, se indagó el conocimiento básico de los adolescentes sobre el uso del condón masculino. A este respecto se preguntó: ¿cuántas veces se puede usar un condón? Los resultados mostraron que $78.0 \%$ del total de adolescentes respondió que una sola vez. En este sentido, $82.6 \%$ de los hombres y $73.2 \%$ de las mujeres respondieron correctamente. En cuanto a la pregunta de si el condón masculino se utiliza para evitar un embarazo o una infección de transmisión sexual, $82.2 \%$ respondió que para ambas situaciones, y el porcentaje de respuesta de uso reportado por sexo fue de $85.1 \%$ para los hombres y $79.1 \%$ para las mujeres. El porcentaje de conocimiento del uso del condón masculino utilizado 
para evitar un embarazo o una infección de transmisión sexual en Sinaloa resultó estar arriba del ámbito nacional (82.2 frente a $78.5 \%$ ) (ENSANUT, 2012).

Del total de adolescentes de 12 a 19 años de edad que han iniciado vida sexual, $33.1 \%$ no utilizó ningún método anticonceptivo en la primera relación sexual, porcentaje mayor al nacional (22.9\%). De los que sí usaron algún método, $64.3 \%$ utilizó condón masculino, cifra menor al porcentaje nacional (72.2 \%). Comparando esta información con la de 2006, se observa que $55.2 \%$ de los adolescentes sinaloenses no utilizó ningún método anticonceptivo en la primera relación sexual, mientras que $35.3 \%$ usó condón masculino (ENSANUT, 2012).

En la última relación sexual, el uso de métodos anticonceptivos muestra que $26.3 \%$ de los adolescentes no utilizó ningún método; mientras tanto, de los que reportaron haber usado alguno, $60.3 \%$ utilizó el condón masculino, dato menor al nacional (66.0 \%). La tendencia de utilización de condón masculino en Sinaloa entre la primera y última relación sexual es similar, como la de los que no utilizaron ningún método. Al comparar esta información con la de 2006, $36.2 \%$ de los adolescentes sinaloenses reportó haber utilizado condón en la última relación sexual (ENSANUT, 2012).

Por lo que se refiere al acceso a condones, $28.4 \%$ de los adolescentes de 12 a 19 años reportó haberlo recibido de forma gratuita en los últimos doce meses, cifra menor a la nacional $(32.7 \%)$. El promedio de condones masculinos recibidos fue de $9.5 \%$. En lo referente al embarazo de adolescentes en Sinaloa, los resultados muestran que $55.7 \%$ de las mujeres de 12 a 19 años con inicio de vida sexual alguna vez han estado embarazadas (ENSANUT, 2012).

En relación a las representaciones sociales (RS), Jodelet señala que cuando las personas hacen referencia a los objetos sociales, los clasifican, explican y evalúan, porque tienen una representación social de ese objeto; y un objeto se representa cuando está mediado por una figura; y es sólo en esa condición que emerge la representación y el contenido correspondiente. Las personas conocen la realidad que les circunda mediante explicaciones 
que extraen de los procesos de comunicación y del pensamiento social. Las representaciones sociales sintetizan dichas explicaciones y, en consecuencia, hacen referencia a un tipo específico de conocimiento que juega un papel crucial sobre cómo la gente piensa y organiza su vida cotidiana: el conocimiento del sentido común (Jodelet, 2006).

La realidad de la vida cotidiana, por tanto, es una construcción intersubjetiva, un mundo compartido. Ello presupone procesos de interacción y comunicación mediante los cuales las personas comparten y experimentan a los otros y a las otras. En esta construcción, la posición social de las personas así como el lenguaje, juegan un papel decisivo al posibilitar la acumulación o acopio social del conocimiento que se transmite de generación en generación. En resumen, el medio cultural en que viven las personas, el lugar que ocupan en la estructura social, y las experiencias concretas con las que se enfrentan a diario influyen en su forma de ser, su identidad social y la forma en que perciben la realidad social. El anterior planteamiento goza del consenso en un amplio sector de quienes realizan investigación en las ciencias sociales (Ibáñez, 1988).

Se trata de acabar con la separación existente entre los procesos y los contenidos del pensamiento social y siguiendo el ejemplo de la antropología y el psicoanálisis, elucidar los mecanismos viendo el contenido que de ellos resulta y deducir los contenidos partiendo de los mecanismos; revertir el rol de laboratorio y el rol de observación, es decir, emprender el estudio de las representaciones sociales en su propio contexto preocupándonos por nuestras realidades. El énfasis en lo colectivo y en la comprensión de la realidad social a partir de su construcción social, son elementos centrales de la teoría de las RS. Por su parte, Moscovici indica que en la interacción sujeto-objeto no existe un solo sujeto, sino que intervienen otros sujetos, a los que el autor les llama Alter (A), que además de relacionarse estrechamente entre ellos y ellas, guardan también íntima relación con el objeto social. Con este planteamiento, Moscovici trasciende de un esquema diádico, donde sujeto (S) y objeto (O) interactúan, para pasar a un esquema triádico donde los otros sujetos también interactúan e inciden en la relación sujeto-objeto (Moscovici, 2000). 
El esquema triádico de Moscovici da supremacía a la relación de sujeto - grupo (otros sujetos), porque: a) Los otros y las otras son mediadores y mediadoras del proceso de construcción del conocimiento y b) La relación de los y las otras con el objeto físico, social, imaginario o real es lo que posibilita la construcción de significados. Esta concepción, a su vez, ilustra sobre la posición epistemológica en la que se inscribe quien estudia las representaciones sociales. En primer término, se parte de que el conocimiento no es sólo comprensible desde la tradicional concepción que señala la existencia de un conocimiento científico y de un conocimiento cotidiano o del sentido común. En esta concepción se comprende el conocimiento como fenómeno o fenómenos complejos que se generan en circunstancias y dinámicas de diversa índole y cuya construcción está multideterminada por relaciones sociales y culturales (Banchs, 1994).

Para la formulación de sus propuestas teóricas estos autores parten de un supuesto básico: la realidad se construye en la vida cotidiana y la sociología del conocimiento debe estudiar los procesos por medio de los cuales se genera el conocimiento. La realidad de la vida cotidiana se me presenta además como un mundo intersubjetivo, un mundo que comparto con otros. Esta intersubjetividad establece una señalada diferencia entre la vida cotidiana y otras realidades de las que tengo conciencia. Estoy solo en el mundo de mis sueños, pero sé que el mundo de la vida cotidiana es tan real para los otros como lo es para mí. En la realidad, no puedo existir en la vida cotidiana sin interactuar y comunicarme continuamente con otros. Sé que mi actitud natural para con este mundo corresponde a la actitud natural de otros, que también ellos aceptan las objetivaciones por las cuales este mundo se ordena, que también ellos organizan este mundo en torno de "aquí y ahora" de su estar en él y se proponen actuar en él" [Entrecomillado en el original] (Berger y Luckmann, 1991, pp. 40$41)$.

El trabajo de Berger y Luckmann aporta tres elementos fundamentales a la propuesta teórica de Moscovici: el carácter generativo y constructivo que tiene el conocimiento en la vida cotidiana. Es decir, que nuestro conocimiento más que ser reproductor de algo preexistente, es producido de forma inmanente en relación con los objetos sociales que conocemos. Que la naturaleza de esa generación y construcción es social, esto es, que pasa por la comunicación y la interacción entre individuos, grupos e instituciones. La 
importancia del lenguaje y la comunicación como mecanismos en los que se transmite y crea la realidad, por una parte, y como marco en que la realidad adquiere sentido, por otra. Estos aspectos contribuyeron de manera significativa en la teoría de las RS. Las aproximaciones al conocimiento cotidiano, considerando seriamente el carácter productor más que reproductor de los significados de la vida social es un elemento claramente visible en la teoría (Elejabarrieta, 1991, p. 259).

Jodelet indica que el campo de representación designa al saber de sentido común, cuyos contenidos hacen manifiesta la operación de ciertos procesos generativos y funcionales con carácter social. Por lo tanto, se hace alusión a una forma de pensamiento social. Las representaciones sociales son la manera en que nosotros, sujetos sociales, aprehendemos los acontecimientos de la vida diaria, las características de nuestro medio ambiente, las informaciones que en él circulan, a las personas de nuestro entorno próximo o lejano. En pocas palabras, el conocimiento "espontáneo", ingenuo que habitualmente se denomina conocimiento de sentido común o bien pensamiento natural por oposición al pensamiento científico (Jodelet, 2006).

El proceso de anclaje, al igual que el proceso de objetivación, permite transformar lo que es extraño en familiar. Sin embargo, este proceso actúa en una dirección diferente al de objetivación. Si lo propio de la objetivación es reducir la incertidumbre ante los objetos operando una transformación simbólica e imaginaria sobre ellos, el proceso de anclaje permite incorporar lo extraño en lo que crea problemas, en una red de categorías y significaciones por medio de dos modalidades: inserción del objeto de representación en un marco de referencia conocido y preexistente. Instrumentalización social del objeto representado o sea la inserción de las representaciones en la dinámica social, haciéndolas instrumentos útiles de comunicación y comprensión (Jodelet, 2006).

Si bien el proceso de anclaje permite afrontar las innovaciones o el contacto con objetos que no son familiares para las personas, hay que advertir que las innovaciones no son tratadas por igual por todos los grupos sociales, lo cual evidencia el enraizamiento social de las representaciones y su dependencia de las diversas inserciones sociales. En efecto, los intereses y los valores propios de los diversos grupos actúan con fuerza sobre los mecanismos de selección de la información, abriendo más o menos los esquemas 
establecidos para que la innovación pueda ser integrada. Si el nuevo objeto que ha aparecido en el campo social es susceptible de favorecer los intereses del grupo, éste se mostrará mucho más receptivo (Jodelet, 2006).

En definitiva, la integración cognitiva de las innovaciones está condicionada tanto por los esquemas de pensamiento ya constituidos como por la posición social de las personas y de los grupos. El proceso de anclaje, a su vez, se descompone en varias modalidades que permiten comprender: cómo se confiere significado al objeto representado, con relación al sentido que se le otorga a la representación, y cómo se utiliza la representación en tanto sistema de interpretación del mundo social marco e instrumento de conducta. Esta modalidad permite comprender cómo los elementos de la representación no sólo expresan relaciones sociales, sino que también contribuyen a constituirlas (Jodelet, 2006).

Cómo opera su integración dentro de un sistema de recepción y la conversión de los elementos de este último relacionados con la representación. Los sujetos se comportan según las representaciones; los sistemas de interpretación proporcionados por la representación guían la conducta. Actuando conjuntamente y por su función integradora, el anclaje y la objetivación sirven para guiar los comportamientos. La representación objetivizada, naturalizada y anclada, es utilizada para interpretar, orientar y justificar los comportamientos (Jodelet, 2006).

Según Moscovici, las RS emergen determinadas por las condiciones en que son pensadas y constituidas, teniendo como denominador el hecho de surgir en momentos de crisis y conflictos. De manera convergente, propone que las representaciones sociales responden a tres necesidades: a) clasificar y comprender acontecimientos complejos y dolorosos; b) justificar acciones planeadas o cometidas contra otros grupos; y c) diferenciar un grupo respecto de los demás existentes, en momentos en que pareciera desvanecerse esa distinción. En suma, causalidad, justificación y diferenciación social. Moscovici infiere tres condiciones de emergencia: la dispersión de la información, la focalización del sujeto individual y colectivo y la presión a la inferencia del objeto socialmente definido (Tajfel, 1999). 
El cuidado humano es el objeto de estudio de la enfermería, esto está constituido por acciones transpersonales e intersubjetivas para proteger, mejorar y preservar la humanidad ayudando a la persona a hallar un significado a la enfermedad, sufrimiento, dolor y existencia y ayudar a otro a adquirir autocontrol, autoconocimiento y auto curación. Sin embargo, las instituciones hospitalarias impregnadas del modelo biomédico y curativo, reducen lo humano a lo biológico alejando el trabajo de las enfermeras(os) de su visión humanista y holística del cuidado (Watson, 2010).

Las instituciones hospitalarias como subsistemas sociales, cumplen la función de socializar a los individuos con pautas, normas establecidas, adaptándolos e integrándolos al sistema, de esta forma el cuidado holístico se va dificultado por las múltiples tareas delegadas de tipo biomédico quedando relegado a acciones como la comunicación eficaz y el interactuar con el paciente y familia en forma cercana, denominado por Watson cuidado transpersonal en dichas acciones valorizadas por los sujetos de cuidado (Watson, 2010).

El concepto de "cuidados integrales" implica que al cuidar hay que atender a la persona en su globalidad, contemplando tanto sus aspectos físicos como psíquicos. Al tener presente que la alteración o desarmonía en cualquiera de sus dimensiones (biológico, psicológica, social, espiritual y unitaria) afectará en mayor o menor grado a las restantes, es obvia la necesidad de cuidar desde una perspectiva global e integral. Cabe resaltar también la importancia de que los cuidados vayan orientados principalmente a la salud. El objetivo fundamental de la enfermería debe ser el fomento a la salud y la prevención de la enfermedad. Cuando esto ya no sea posible, los cuidados deben de ser dirigidos hacia la resolución del problema y la rehabilitación o fomento de conductas adaptivas, o hacia la ayuda al "bien morir" de la persona (Novel, 2005).

Los modelos y teorías de enfermería se fundamentan en una visión humanista del cuidado, por ejemplo Watson refiere que el cuidado es para la enfermería su razón moral, no es un procedimiento o una acción, el cuidar es un proceso interconectado, intersubjetivo, de sensaciones compartidas entre la enfermera(o) y el sujeto de cuidado. El cuidado humano debe basarse en la reciprocidad y debe tener una calidad única y auténtica. La enfermera(o) es la llamada a ayudar al paciente a aumentar su armonía dentro de la mente, del cuerpo y del alma, para generar procesos de conocimiento de sí mismo. Desde este punto de vista, el 
cuidado no sólo requiere que la enfermera(o) sea científica, académica y clínica, sino también un agente humanitario y moral, como copartícipe en las transacciones de cuidados humanos (Watson, 2010).

A través de estudios transculturales, Leininger realiza los primeros intentos para clarificar y conceptualizar la noción del cuidado: el cuidado es para enfermería el dominio central del cuerpo de conocimiento y las prácticas. Afirma que el constructo cuidado se ha manifestado durante millones de años como fundamental en el crecimiento y supervivencia de los seres humanos. El cuidado permite a la especie humana vivir y sobrevivir bajo las más adversas condiciones ambientales, sociales, económicas y políticas. Los antecedentes del cuidado ya se remontaban a la mitología romana, en que el cuidado es quien crea al hombre y lo protege. De esto se deriva que la acción de cuidar es, entonces, algo propio del ser humano y revela su íntima constitución. Leininger considera que el cuidado es de carácter universal, pero son los procesos, métodos y técnicas con los que se desarrollan los que varían culturalmente, es así que en la cultura anglosajona se distingue entre los términos de "careí" y "caring", "carê̂" es la acción de cuidar y "caring" incorpora además un sentido e intencionalidad humanitaria en enfermería (Watson, 2010).

Otro aspecto a considerar por teóricos de enfermería como Leininger es establecer la diferencia que existe entre curación y cuidados, donde agrega: los procesos de curar y cuidar presentan diferencias en su esencia y en sus características principales; no puede existir curación sin cuidados, pero pueden existir cuidados sin curación. Las diferencias ontológicas entre las actividades de curar y las de cuidar, de acuerdo a Medina, se basan en la disimilitud de la perspectiva epistemológica y filosófica de la que ambas parten. Los aspectos biomédicos y curativos se basan en una perspectiva analítica, empírica y experimental, reduciendo lo humano a lo biológico, el ser humano es una enfermedad u objeto de estudio (Watson, 2010).

En cambio, el cuidado de origen ancestral tiene una mirada diferente, ve al sujeto como enfermo-sujeto, antes que su enfermedad-objeto, permitiendo que el sufrimiento que puede ser aliviado no sea ocultado en la profundidad biológica del organismo. Los cuidados reducen las distancias entre los tratamientos y el sentido de la enfermedad, por lo cual enfermería se basa en una visión holística, biopsicosocial, centrándose en las respuestas a 
los procesos mórbidos y de salud. Otros autores como Colliere, establecen que este saber ancestral de enfermería, ha estado oculto por la hegemonía de la racionalidad técnica, encarnada en el saber médico, que ha prevalecido en los sistemas de salud. De acuerdo a esta autora, enfermería viene desarrollando tres tipos de actividades diferentes dentro de estas instituciones: las actividades que dependen directamente de la decisión e iniciativa de la enfermera (Watson, 2010).

En contraparte, la visión humanista del cuidado de Watson nos habla de la importancia de la visión de los cuidados de Enfermería en los sistemas hospitalarios; el cuidado debe adquirir una dimensión profunda que va más allá de una simple técnica, de planificar una atención, de recibir un turno o la de una educación rutinaria día a día; es estar ahí con el otro, es compartir sus sentimientos y sus emociones (Watson, 2010).

Otro aspecto importante que se debe de tomar en cuenta en el cuidado es la perspectiva de género. La disciplina que primero utilizó la categoría género para establecer una diferencia con el sexo fue la psicología, en su vertiente médica. Robert Stoller (Sex and Gender, 1968) estudió los trastornos de la identidad sexual, examinando casos en los que la asignación de sexo falló, ya que las características externas de los genitales se prestaban a confusión. Tal es el caso de niñas cuyos genitales externos se han masculinizado, por un síndrome adrenogenital; o sea, niñas que, aunque tienen un sexo genético (xx), anatómico (vagina y clítoris) y hormonal femenino, tienen un clítoris que se puede confundir con pene (Lama, 2008).

El papel (rol) de género se forma con el conjunto de normas y prescripciones que dictan la sociedad y la cultura sobre el comportamiento femenino o masculino. Aunque hay variantes de acuerdo con la cultura, la clase social, el grupo étnico y hasta el nivel generacional de las personas, se puede sostener una división básica que corresponde a la división sexual del trabajo más primitiva: las mujeres paren a los hijos, y por lo tanto, los cuidan: ergo, lo femenino es lo maternal, lo doméstico, contrapuesto con lo masculino como lo público. La dicotomía masculina-femenina, con sus variantes culturales (del tipo yang y yin), establece estereotipos las más de las veces rígidos, que condicionan los papeles y limitan las 
potencialidades humanas de las personas al estimular o reprimir los comportamientos en función de su adecuación al género (Lama, 2008).

La perspectiva de género implica reconocer que una cosa es la diferencia sexual y otra son las atribuciones, ideas, representaciones y prescripciones sociales que se construyen tomando como referencia esa diferencia sexual. Todas las sociedades estructuran su vida y construyen su cultura en torno a la diferencia sexual. Esta diferencia anatómica se interpreta como una diferencia sustantiva que marcará el destino de las personas. Lo lógico, se piensa, es que si las funciones biológicas son tan dispares, las demás características morales, psíquicas también lo habrán de ser (Lama, 2008).

En México, la población adolescente de entre 10 y 19 años de edad representa $23.2 \%$ del total, es decir, casi 21 millones de personas. La edad promedio para el inicio de relaciones sexuales es de 16 años. Los varones se inician antes que las mujeres con una diferencia de uno o dos años. Asimismo, $76 \%$ de los varones solteros y $35 \%$ de las mujeres solteras menores de 20 años declaran haber tenido relaciones sexuales. Los hombres informan haber tenido su primera relación sexual con una amiga, mientras las mujeres dicen que se inician con su novio. La agenda de la Conferencia Internacional de Población y Desarrollo del Cairo (1994) reconoce el derecho de los/las adolescentes a la educación sexual y a servicios de salud adecuados para ellos (SSR, SSA, 2001).

Según la Organización Mundial de la Salud (OMS), la adolescencia es una de las etapas de la vida y uno de los grupos de población, que se ubica entre los 10 y 19 años de edad (definición cronológica). Se inicia con la pubertad, la cual se caracteriza por cambios biológicos y corporales como son el desarrollo de los caracteres sexuales secundarios y la adquisición de la capacidad reproductiva (definición biológica). Los cambios emocionales más importantes de esta etapa son: la búsqueda de identidad, la separación de los padres, el duelo por la infancia y el fortalecimiento del yo (definición psicológica). Se concibe como un periodo de aplazamiento y preparación para las responsabilidades de la vida adulta: encontrar empleo, iniciar la vida en pareja o convertirse en padre o madre, que se definen de acuerdo a cada cultura (definición sociocultural) (SSR, SSA, 2001). 
La salud reproductiva supone un estado de completo bienestar físico, mental y social en lo que respecta a todo lo relacionado con el sistema reproductivo, sus funciones, procesos, y no simplemente a la ausencia de enfermedad o debilidad. La salud reproductiva implica la capacidad de disfrutar de una vida sexual satisfactoria, sin riesgos y de procrear, la libertad para decidir hacerlo o no hacerlo, cuándo y con qué frecuencia. El hombre y la mujer tienen el derecho a obtener información y acceso a métodos seguros, eficaces, asequibles y aceptables para la regulación de la fecundidad, así como el derecho a recibir servicios adecuados de atención a la salud que permitan el embarazo y el parto sin riesgo y con las mayores posibilidades de tener un hijo saludable. Organización Mundial de la Salud (SSR, SSA, 2001).

Los derechos sexuales forman parte de los derechos humanos básicos, es decir, lo que tenemos las personas por el hecho de serlo. Estos derechos se refieren al respeto de la integridad física del cuerpo humano, al derecho a la información y a los servicios de salud sexual, al derecho a tomar decisiones sobre la propia sexualidad y la reproducción. Los y las adolescentes deberán conocer en primer lugar que tienen derechos sexuales y reproductivos, y que al igual que los derechos humanos, forman parte de sus garantías individuales y, por lo tanto, que pueden solicitar información sobre sexualidad y servicios de salud sexual y reproductiva accesibles, sin temor a ser sancionados (SSR, SSA, 2001).

Todos los adolescentes del mundo, sin importar sexo, religión, color, orientación sexual o habilidad física o mental, tienen los siguientes derechos como seres sexuales: el derecho a ser ella/el mismo, libre de tomar sus propias decisiones de expresar lo que piensa, de disfrutar de la sexualidad, de estar seguro/a, de escoger casarse (o no casarse) y de planear una familia. El derecho a estar informado sobre sexualidad, anticonceptivos, infecciones de transmisión sexual ITS y VIH/SIDA y sobre sus derechos sexuales. El derecho a estar protegido de embarazos no planeados, ITS, VIH/SIDA y de abusos sexuales. El derecho a tener servicios médicos confidenciales, a precios accesibles, de buena calidad y respetuosos. El derecho a participar en la planeación de programas juveniles, tomar parte en reuniones y seminarios y tratar de influir en los gobiernos, por los medios apropiados (SSR, SSA, 2001). 


\section{Material y método}

El diseño metodológico de esta investigación consistió en un estudio cualitativo descriptivo interpretativo de orientación procesual, haciendo uso de técnicas analíticas, a partir de los resultados y especificidad de los participantes de la investigación. Concretamente, contempló la formulación de categorías de análisis iniciales, que se identificaron durante el proceso de construcción teórica y análisis de resultados de la técnica de redes de asociaciones (De la Rosa, 1995).

\section{Resultados y discusión}

En este apartado se presentan los resultados obtenidos en las entrevistas realizadas con el principio de saturación teórica relacionados con el tema en estudio, a través de la entrevista individual a profundidad y la observación participante, con el diálogo establecido entre los investigadores y las(os) estudiantes de enfermería en Culiacán, Sinaloa. Después de realizar la recolección de los datos del enfoque procesual de las representaciones sociales de los métodos anticonceptivos, el análisis de la información con los participantes durante la entrevista y a través del análisis de contenido, surgieron los significados de una categoría con cinco subcategorías.

Categoría I: Representación social de los métodos anticonceptivos femenino y masculino, con cinco subcategorías: 1) Condón masculino, 2) Es responsabilidad del hombre y la mujer protegerse, 3) Pastillas/DIU, 4) Píldora de emergencia, 5) Coito interrumpido/Condón.

Podemos entender las representaciones sociales como formas de conocimiento a partir de las cuales los individuos comprenden, perciben y organizan los fenómenos de la realidad social (Moscovici, 2002). El análisis de las representaciones sociales ha llegado a ser un objeto de estudio privilegiado para recoger las formas de construcción cognitiva y simbólica con que los sujetos abordan la realidad. La sexualidad, como dimensión del ser humano, de hecho es una representación personal pero también social que se organiza en estructuras mentales individuales y colectivas como un dominio autónomo y protegido al que subyace un saber subjetivo colectiva y culturalmente compartido. 
Se coloca así en evidencia la articulación entre: las vivencias privadas, las prácticas individuales y colectivas, y los saberes y tradiciones que les sirven de soporte. En otras palabras, un movimiento que va desde el sujeto a la sociedad y desde la sociedad hacia el sujeto: una construcción cognitiva, una elaboración psicológica y social de la realidad, hecha en la interacción con los otros contribuyendo a producir un sentido común, una visión común a un segmento social y cultural dado: en el caso de nuestro estudio. Esta interacción con los otros se da en el espacio de la conversación, experiencia humana inter subjetiva orientada a hacer sentido de la experiencia de la realidad social. La conversación es un espacio inmediato disponible para la producción e intercambio de representaciones (Moscovici, 2002).

Las representaciones, en su productividad de sentidos comunes a través de la conversación, se sitúan en el seno de la experiencia de la vida cotidiana, en el lugar de emergencia de la experiencia moral. "La moral, en cuanto experiencia de la vida cotidiana, posee una dimensión de comunicación y reconocimiento que tienen su expresión en los sentidos comunes de los grupos y comunidades: Moral significa aquí experiencia moral, aquella en y por la que tú y yo nos reconocemos y nos comunicamos en las múltiples interacciones propias de una convivencia real". Las representaciones en el campo de la sexualidad no son ajenas a la producción de moralidades cotidianas, en su dimensión de hábitos sociales. Este es un lugar especialmente sensible cuando se trata de intervenciones comunicacionales en función de la formación de hábitos en la sexualidad de los sujetos (Mercadeo social del condón, 2005).

Los resultados del enfoque procesual de las representaciones sociales de los métodos anticonceptivos mostraron que la mayoría de los estudiantes de ambos sexos realizaron asociaciones vinculadas a la categoría de cuidado de la salud sexual y reproductiva en los métodos anticonceptivos femenino y masculino. Se encontró que el núcleo central de dicha representación se forma en torno al cuidado con condón masculino acompañado de ejes periféricos de la representación protectores de la salud, tales como el consenso de que es responsabilidad del hombre y la mujer protegerse por igual y la combinación de métodos 
como las pastillas/DIU. También se encontraron diferencias de género, en el caso de las mujeres un nivel significativo representacional pero también periférico es la práctica del uso de la píldora del día siguiente. Y en el caso de los hombres combinar la práctica del coito interrumpido/condón. También hay que tener en cuenta la presencia de otros ejes periféricos que sin ser los de mayor importancia, en su conjunto muestran particularidades de la representación. DIU, pastillas, utilización de métodos hormonales, salpingoclasia y relaciones no vaginales.

De hecho, en las redes de asociaciones que se presentan en la configuración del núcleo central de la representaciones sociales en al análisis de los discursos de las entrevistas se tuvieron en cuenta las categorías determinadas en las redes de asociaciones. El análisis de contenido de las entrevistas posibilitó destacar el núcleo central, lo cual también se observa en los discursos siguientes cuando los entrevistadores preguntan:

¿Qué significan para usted los métodos anticonceptivos?

\section{Discursos femeninos}

- Una manera de control de la natalidad. (F-1)

- Son métodos que se utilizan para llevar una planificación familiar planeada. (F-2)

- Un método necesario para planificar la familiar y tener la que uno desea. (F-3)

- Son la forma de planificación familiar y evitar embarazos no deseados. (F-4)

- Son muy importantes ya que previenen embarazos y, en algunos casos, enfermedades de trasmisión sexual. (F-5)

- Pues son métodos que nos ayudan a las mujeres y hombres a tener una sexualidad activa responsable. (F-6)

- Son métodos de anticoncepción que impiden tanto la concepción de embarazos como las enfermedades. (F-7)

- Son barreras que pueden ayudar a prevenir embarazos no deseados, así como también diversas enfermedades de transmisión sexual. (F-8)

- Una buena herramienta para planear o espaciar embarazos y prevenir enfermedades. (F-9) 
- Los métodos anticonceptivos, son herramientas de barrera que se utilizan para prevenir embarazos y enfermedades de transmisión sexual, por ejemplo, el condón, DIU, pastillas, etcétera. (F-10)

Los discursos de las estudiantes de enfermería enfatizan que los métodos anticonceptivos son dispositivos que se utilizan para llevar a cabo la planificación familiar y que además ayudan a prevenir embarazos no deseados, y en el caso del uso del condón previene el contagio de enfermedades de transmisión sexual.

\section{Discursos masculinos}

- Son los métodos por los cuales se trata de que la mujer no quede embarazada. (M-1)

- El cuidarnos de un embarazo no deseado y prevenir enfermedades de transmisión sexual. (M-2)

- Protegerse y no tener embarazos no deseados. (M-3)

- Métodos para proteger a personas sexualmente activas de enfermedades de transmisión sexual y embarazos no deseados. (M-4)

- Protección contra enfermedades y embarazos no deseados. (M-5)

- Son todos aquellos métodos que nos sirven para evitar un embarazo y cuidarnos de las enfermedades de transmisión sexual. (M-6)

- Son los métodos que nos sirven para la planificación familiar. (M-7)

- Son barreras que se utilizan para cuidarse de enfermedades y de embarazos no deseados. (M-8)

- Son los métodos que se utilizan para prevenir embarazos no deseados y enfermedades de transmisión sexual. (M-9)

- Dispositivos para evitar un embarazo. (M-10)

Los discursos de algunos estudiantes de enfermería sobre los métodos anticonceptivos también enfatizan que mediante su uso nos pueden ser útiles para cuidar nuestra salud sexual y reproductiva al prevenir embarazos no planeados, y además también algunos métodos como el condón nos permiten protegernos de enfermedades de transmisión sexual. 
Es decir, para las estudiantes de enfermería los métodos anticonceptivos significan: una manera de control de la natalidad, una planeación familiar que permite también prevenir los embarazos no deseados y en algunos casos también las enfermedades de transmisión sexual. Además, el uso de métodos anticonceptivos ayuda tanto a mujeres como a hombres a ejercer su sexualidad de manera sana y responsable. También se conciben como herramientas para planear o espaciar embarazos deseados. Por otra parte, en el caso de los estudiantes de enfermería se representan los métodos anticonceptivos como herramientas que nos permiten evitar un embarazo no deseado, así como planear los hijos que se desean. También permiten ejercer una sexualidad sana y responsable: el uso del condón evita embarazos y algunas enfermedades de transmisión sexual.

Los entrevistadores vuelven a preguntar:

¿En su opinión que método anticonceptivo recomendaría a un amigo o amiga? ¿Por qué?

\section{Discursos femeninos}

- Condón o dispositivo son los más seguros. (F-1)

- Condón, porque es un método anticonceptivo que previene las enfermedades de transmisión sexual. (F-2)

- Condón, ya que con él se cuida de enfermedades de trasmisión sexual y de los embarazos no deseados. (M-3)

- El condón porque te cuida de embarazos y puede ayudar un poco a evitar alguna enfermedad. (F-4)

- Condón, porque es más confiable siempre y cuando se utilice adecuadamente. (F-5)

- El DIU, porque es más cómodo. (F-6)

- El implante hormonal junto con el condón, porque yo lo utilizo desde hace cuatro años y sí funciona. (F-7)

- El condón debido a que está al alcance de todos y puede proteger contra enfermedades de transmisión sexual. (F-8)

- El condón y el DIU, porque creo que en el matrimonio el DIU es cómodo y seguro. (F-9) 
- Con condón, porque es un método $99.99 \%$ seguro para prevenir embarazos y enfermedades de transmisión sexual. (F-10)

\section{Discursos masculinos}

- El preservativo es el más confiable y menos dañino. (M-1)

- El condón, porque está más a la mano de cualquier persona. (M-2)

- El condón porque es uno de los más efectivos. (M-3)

- Con condón. Tiene 99 \% de seguridad para cuidarse de las enfermedades de trasmisión sexual y embarazos no deseados. (M-4)

- El condón es el más práctico y también es el más efectivo para cuidarse de una manera correcta. (M-5)

- El uso del condón, ya que es fácil de obtener y está al alcance de todos. (M-6)

- A las mujeres el implante ya que es más efectivo para no embarazarse y al hombre el condón para no enfermarse. (M-7)

- El condón es uno de los métodos más seguros y accesibles. (M-8)

- El condón y las pastillas. (M-9)

- El condón porque es el método más común y más sencillo de usar. (M-10).

Las(os) estudiantes de enfermería, tanto mujeres como hombres, utilizan diferentes métodos anticonceptivos para cuidar su salud sexual y reproductiva, tales como el condón masculino, pastillas en combinación con el DIU, la píldora de emergencia y en ocasiones el coito interrumpido, y consideran al condón masculino como un método fácil, seguro, efectivo y preventivo; se ha demostrado que el condón masculino de látex es el único método anticonceptivo que protege contra la transmisión de casi todos los tipos de ITS, incluyendo un alto grado de protección contra la infección por el VIH (Secretaría de Salud de México, 2002).

Tanto mujeres como hombres se cuidan con anticonceptivos como condón, pastillas, DIU y abstinencia. "Las representaciones sociales constituyen una forma de conocimiento socialmente elaborada, que se establece a partir de la información que recibe el individuo 
de sus experiencias y modelos de pensamiento compartidos y transmitidos entre jóvenes" (Pérez, A).

La estructura representacional del cuidado de la salud sexual y reproductiva en los métodos anticonceptivos masculino y femenino se organizó alrededor de un núcleo figurativo que incluye varias subcategorías. El uso del condón masculino como método favorito por excelencia, acompañado de la responsabilidad que deben asumir ambos sexos para cuidarse mutuamente, pastillas y DIU, la píldora de emergencia. Una última subcategoría sólo en el caso de los hombres fue alternar el coito interrumpido con el uso del condón.

A continuación se presentan los desarrollos analíticos en las categorías antes señaladas.

\section{2) Es responsabilidad de ambos sexos protegerse}

Se pregunta: ¿quién debe de cuidarse más en una relación sexual, el hombre o la mujer?

\section{Discursos femeninos}

- La mujer se debe cuidar de no embarazarse y no contraer alguna enfermedad venérea $(\mathrm{F}-1)$

- La mujer siempre lleva la de perder, por eso ella es quien se tiene que cuidar más, porque ella se puede embarazar (F-2)

- Las mujeres debemos cuidarnos y saber bien con quién te metes, es decir, conocer bien a la persona $(\mathrm{F}-3)$

\section{Discursos masculinos}

- Los dos se deben de cuidar ya que está de por medio la salud y, sobre todo, tratar de no embarazar a la pareja (M-1)

- La mujer se debe de cuidar más porque ella es quien se queda con los hijos si no le responde la pareja $(\mathrm{H}-2)$

- Las mujeres, ya que pueden quedar embarazadas (H-3) 
Las(os) estudiantes de enfermería, tanto mujeres como hombres, coinciden en que las mujeres son las que deben de llevar la responsabilidad del cuidado de su salud sexual y reproductiva y la de la pareja, ya que socialmente se configura la representación de que son ellas las que quedan embarazadas y son ellas las de la responsabilidad de criar solas a los hijos si no cuentan con el apoyo de la pareja. En segundo término está su salud, que no se visualiza sino hasta que se previene el embarazo. Los cuerpos se constituyen histórica y culturalmente, y responden a las necesidades, ideologías, pensamientos y percepciones idealizadas de una sociedad, para conducirse con una sexualidad fundamentada en principios morales, no sólo en cuanto a la estructura fisiológica, sino también la personalidad y las apreciaciones sobre las que el individuo dirige o actúa constantemente en su vida.

En tanto, los estudios de género y sexualidad refieren que las construcciones simbólicas de los roles desempeñados por mujeres y hombres, son aprendidas por los individuos y las relacionan a lo largo de su vida, admitiéndolas como una forma normal de vivir su sexualidad (Martínez, 2005).

Weeks (1998) menciona que los individuos no sólo son portadores de un cuerpo sexuado, sino que también su destino está ligado a los constructos culturales de la sociedad, comenzando por el lugar y el tiempo en que viven y crecen. Con base en esta postura, la sexualidad determina en el hombre cómo debe ser y qué hacer con el cuerpo que posee, en función de los aspectos culturales e históricos que establecen atribuciones y particularidades sobre el cuerpo sexuado (Martínez, 2005).

La sexualidad es manejada de diferente manera en hombres y mujeres, partiendo de la edad, el sexo, los roles de género y el contexto. Este es el caso del hombre universitario, cuya personalidad está influenciada por sus vivencias, experiencia familiar y los aspectos culturales, de tal forma que ser parte de una institución de nivel superior, lo hace diferente de los demás jóvenes. Estar en este nivel educativo significa un logro en el ámbito social, que es de gran importancia no sólo en lo individual sino también en su interacción con los demás. Es así que el individuo muestra energía y entusiasmo por culminar un ciclo educativo, así como capacidad para desarrollar nuevas habilidades y metas que consideraba 
desconocidas. Poco a poco, a través de sus relaciones dentro y fuera de la universidad, se concebirá preparado para asumir un comportamiento "como hombre” (Martínez, 2005).

Consciente o inconscientemente se tiene una necesidad de pertenencia, además de que sus pares lo identifiquen al ser incluido a un grupo social, debido a que un colectivo define a los hombres de los demás y esto les permite demostrar ser más interesantes, atrevidos, conocedores y experimentados de la vida, dando esta idea y apariencia hacia las mujeres universitarias. Por ello es común identificar que a su ingreso a la universidad los chicos tienen una forma de vestir, pensar y desarrollarse en esta etapa, pero al ir transcurriendo el tiempo algunos comienzan a cambiar su forma de vestir, expresarse y opinar sobre las circunstancias de la vida. Esto se da como respuesta a las redes sociales que se desarrollan dentro y fuera de la institución educativa, y por los contenidos vistos de manera profesional (Pérez et al., UNAM, 2009).

\section{3) Pastillas en combinación con el dispositivo intrauterino}

Como se observa en los discursos siguientes, cuando los entrevistadores preguntan: ¿además del condón, que otro método anticonceptivo recomiendas y por qué?

\section{Discursos femeninos}

- Las pastillas, porque son muy efectivas para prevenir el embarazo (F-1)

- El DIU ya que es fácil de utilizar y no conlleva ningún riesgo para la salud (F-2)

- Yo utilizo el DIU desde que tuve a primer hijo y me ha funcionado muy bien (F-3)

- Las pastillas son fáciles de seguir y son $100 \%$ efectivas (F-4)

- Las pastillas, porque es fácil y si se te olvida tomar una no pasa nada (F-5)

- El DIU porque no conlleva ningún riesgo y es muy efectivo (F-6)

Las estudiantes de enfermería coincidieron en utilizar las pastillas anticonceptivas como método de control de la natalidad, seguido por la utilización del DIU por ser métodos altamente efectivos y que no conllevan ningún riesgo para su salud sexual. 


\section{4) Píldora de emergencia}

Los entrevistadores preguntan: ¿recomendarías el uso de la píldora de emergencia para prevenir un embarazo no deseado?

\section{Discursos femeninos}

- Claro que sí, dicen que es muy efectiva dentro de las primeras 24 horas después de la relación sexual $(\mathrm{F}-1)$

- Sí, por ser muy efectiva y fácil de utilizar, además la recomiendan mucho en la televisión (F-2)

- Sí, es mejor utilizarla cuando no se usó condón por parte de la pareja para no quedar embarazada (F-3).

Las estudiantes asumen que es mejor utilizar la píldora del día siguiente para prevenir un embarazo no deseado cuando la mujer o la pareja por algún motivo no se cuidó y que es mejor utilizar este último recurso antes que quedar embarazada.

Esto se refiere al uso de píldoras anticonceptivas para prevenir el embarazo poco después de haber tenido un coito sin protección. La anticoncepción de emergencia no se ha diseñado como una forma habitual de anticoncepción. Después del uso, se debe iniciar o continuar un método habitual si no se desea el embarazo. Las píldoras anticonceptivas de emergencia o PAE, son un régimen especial de píldoras sólo de progestina o de anticonceptivos orales combinados (Introducción a los métodos anticonceptivos/SSA, 2002).

\section{5) Coito interrumpido combinado con la utilización del condón}

Los entrevistadores preguntan: ¿además del condón, qué otro método anticonceptivo recomiendas y por qué?

\section{Discursos masculinos}

- Más que otro método estaría bien lo natural porque no se siente lo mismo (H-1)

- El condón está bien, pero de vez en cuando hay que hacerlo sin condón tomando las precauciones adecuadas $(\mathrm{H}-2)$ 
- No hay mejor cosa que lo natural, pero por lo del embarazo y las enfermedades por eso se utiliza el condón.

Los estudiantes mencionan que el coito interrumpido es una práctica muy frecuente en la consumación del coito, a pesar de que saben que es una conducta de alto riesgo para que se presente un embarazo o una enfermedad de transmisión sexual. La creencia de que "no se siente lo mismo", está aún muy arraigada en las prácticas machistas del estereotipo dominante.

El coito interrumpido y la abstinencia periódica son métodos anticonceptivos que las parejas siempre tienen a su disposición, no cuestan nada y no tienen ningún efecto secundario. También conocido como "coitus interruptus', requiere que el pene se extraiga de la vagina antes de la eyaculación para impedir que haya contacto entre los espermatozoides y el óvulo. La eficacia anticonceptiva es similar a la de los métodos de barrera, con tasas de embarazo de aproximadamente $4 \%$ con el uso perfecto y de por lo menos $19 \%$ con el uso típico durante el primer año. Incluso cuando este método se usa correctamente, todavía hay posibilidades de embarazo ya que el líquido pre-eyaculatorio puede contener espermatozoides. El uso correcto y sistemático del coito interrumpido requiere que el hombre sea disciplinado y tenga control de sí mismo para que extraiga el pene de la vagina de su compañera antes de la eyaculación (Introducción a los métodos anticonceptivos/SSA, 2002). 


\section{Conclusiones}

Los procesos de anclaje y de objetivación de las representaciones sociales de los métodos anticonceptivos se centran principalmente en la utilización del condón masculino en ambos sexos como método primigenio para el control de la natalidad y prevención de enfermedades de transmisión sexual. Las políticas públicas en materia de control de la natalidad están centradas principalmente en la corporeidad femenina (pastillas, inyecciones, DIU, entre otros). Y prácticamente el condón masculino está en el centro de la representación social de ambos sexos como método de primera elección. Se encontró que el núcleo central de dicha representación se forma en torno al cuidado con condón masculino acompañado de ejes periféricos de la representación protectores de la salud, tales como el consenso de que es responsabilidad del hombre y la mujer protegerse por igual y la combinación de métodos como las pastillas/DIU. También se encontraron diferencias de género, en el caso de las mujeres un nivel significativo representacional pero también periférico es la práctica del uso de la píldora del día siguiente. Y en el caso de los hombres combinar la práctica del coito interrumpido/condón. También hay que tener en cuenta la presencia de otros ejes periféricos que sin ser los de mayor importancia, en su conjunto muestran particularidades de la representación DIU, pastillas, utilización de métodos hormonales, salpingoclasia y relaciones no vaginales. 


\section{Bibliografía}

Banchs, M. (1994). Desconstruyendo una desconstrucción: Representation. Threads of discussion, Electronic Version 3. Peer Reviewed Online Journal. 1- 20. www.swp.uni-linz.ac.at/content/psr/psrindex.htm

Berger y Luckmann (1991). La construcción social de la realidad, Buenos Aires, Argentina, Amorrortu editores.

Candreva, A. et al. (2004). Cuidado de la salud: El anclaje social de su construcción. Argentina, Universidad Nacional de la Plata.

Collazos (2012). Representaciones sociales de la salud sexual de adolescentes sordos y oyentes en la ciudad de Bogotá. Pensamiento Psicológico, vol. 10, núm. 2, 2012, pp. 35-47. Cali, Colombia. Pontificia Universidad Javeriana.

De Rosa, A. (1995). Le réseau d'associations comme méthode d'étude dans la recherché sur les representations sociales: structure, contenus et polarité du champ sémantiqe. Les cahiers internationaux de psychologie sociale, 28, 97- 123.

Elejabarrieta, F. (1991). Las representaciones sociales. Bilbao, España, En Echevarría, A. Psicología social sociocognitiva.

Fernández, P., et al. (2014). Dinámica demográfica 1990-2010 y proyecciones de población 2010-2030. 2014, de CONAPO Sitio web: www.conapo.mx.

Fundación Mexicana para la Planeación Familiar (1995). Perspectiva hacia el siglo XXI: la nueva cultura de la salud sexual. México, Mexfam.

Hernández, R. et al. (2014). Metodología de la investigación. Lima, Perú, McGraw- Hill.

Ibáñez, T. (1988). Ideologías de la vida cotidiana. Sendai, Barcelona, España.

INE (2015). Contexto geográfico de la entidad federativa. México, DF. En Memoria del Proceso Electoral Federal 2005-2006 (5).

Instituto Nacional de Salud Pública. Encuesta Nacional de Salud y Nutrición (2012). Resultados por entidad federativa, Sinaloa. Cuernavaca, México.

Jodelet, D. (2006). Formación de representaciones sociales. V. (comp.) Les savoirs du quotidien. Francia, Editorial Haas.

Lamas, M. (2008). Cuerpo: diferencia sexual y género, México, DF, Taurus. 
Lerner, S. (2009). Salud reproductiva y condiciones de vida en México. Edición del Colegio de México.

Marriner A, (2005). Modelos y teorías en enfermería. Madrid, Elsevier.

Martínez, A. (2005). Educación y prevención del SIDA. Anales de Psicología, 21, 8.

MEXFAM (1995). Fundación mexicana para la planeación familiar, México.

MINSA/UNFPA (2009). Plan de Mercadeo Social del Condón 2009-2011. Lima, IES.

Moscovici, S., et al. (2000). Psicología Social II: pensamiento y vida social. Psicología social y problemas sociales. México, Paidós.

Novel, M. et al. (2005). Enfermería psicosocial y salud mental, Barcelona, España Editorial: Elsevier Masson.

ONU (1994). Conferencia internacional sobre población y desarrollo. El Cairo, Egipto.

OPS (2000). Promoción de la salud sexual recomendaciones para la acción, Antigua Guatemala, Asociación Mundial de Sexología.

Pérez D. et al., (2011). Libertad y responsabilidad en la vida sexual de los jóvenes universitarios. México, DF, Revista Digital Universitaria, 12, 19.

SSA (2002). La salud sexual y reproductiva en la adolescencia: un derecho a conquistar. México, DF: Dirección General de Salud Reproductiva.

SSA (2002). La salud sexual y reproductiva en la adolescencia: un derecho a conquistar, México DF.

SSA (2002). Introducción a los métodos anticonceptivos: Información general. México, DF: Dirección General de Salud Reproductiva.

Tajfel et al. (1999). Social categorization and intergroup behaviour. European Journal of Social Psychology.

Watson J. (2010). Filosofía y teoría de los cuidados humanos en enfermería en: Riehl Sisca J, editor. Modelos conceptuales de enfermería. Barcelona: Doyma; pp. 179-92.

Silvana Da Rosa, Ana María (1995). Técnica de Redes de Asociaciones. 